
internationales

vol. 30 - $n^{\circ} 2$ | 2014

Composer (avec) la frontière. Passages, parcours migratoires et échanges sociaux

\title{
Passages de frontières des Palestiniens du Liban : de refuges en migrations
}

Crossing Borders: Palestinians Refugees in Lebanon from Refugees to Migrants

Cruces de fronteras de los palestinos de Líbano: de refugios en migraciones

\section{Nicolas Puig}

\section{(2) OpenEdition} Journals

\section{Édition électronique}

URL : https://journals.openedition.org/remi/6882

DOI : $10.4000 /$ remi.6882

ISSN : $1777-5418$

Éditeur

Université de Poitiers

Édition imprimée

Date de publication : 1 juin 2014

Pagination : 49-67

ISBN : 979-10-90426-22-1

ISSN : 0765-0752

\section{Référence électronique}

Nicolas Puig, « Passages de frontières des Palestiniens du Liban : de refuges en migrations », Revue européenne des migrations internationales [En ligne], vol. 30 - n² | 2014, mis en ligne le 01 juin 2017, consulté le 15 avril 2022. URL : http://journals.openedition.org/remi/6882 ; DOI : https://doi.org/ 10.4000/remi.6882 


\section{Passages de frontières des Palestiniens du Liban : de refuges en migrations}

\section{Nicolas Puig'}

"Ici pour nous laisser passer, on doit vérifier notre carte d'identité. Une fois qu'on a su qu'on vient du camp de Nahr al-Bared on nous fouille, on nous arrête pendant une heure. On a besoin d'une autorisation pour pouvoir entrer chez soi, dans son camp et on nous fait des problèmes, c'est terrible. Si on commet une faute, on nous accuse d'être Palestiniens. On n'a rien ici, ni droits, ni rien d'autre " (Najdé, Nahr al-Bared, Liban, 2011).

" Je suis parti à Athènes ; c'est-à-dire, que dire... L'enfermement d'une prison, c'était

l'équivalent d'une grande prison; Athènes était comme une grande prison. L'anarchie totale, mais tu ne pouvais pas travailler, tu ne peux pas travailler; tu ne connais personne avec qui tu peux travailler. [...] Mon avion était, disons à 11 heures, je suis arrivé au Liban

à 13 heures et quelques. Je me suis dit: "c'est la première fois que je prends l'avion". Mon Dieu, toute cette souffrance que j'ai endurée à l'aller, tout ça, nous avions frôlé la mort en arrivant en Grèce ; et à la fin, en $1 \mathrm{~h}$ 45, tu es au Liban ; vraiment, c'est quelque chose! Sérieusement, c'est perturbant » (Baha, Nahr al-Bared, Liban, 2011).

Cet article $^{2} \mathrm{~s}^{\prime}$ intéresse à une population rejetée à la marge de la société qui I'accueille depuis 1948. À cette date intervient I'exode de près de 700000 Palestiniens dont 100000 se fixent durablement au Liban. Pris en charge par un organisme de I'ONU dédié à leur cas, I'UNRWA (United Nation Relief and Works Agency), ils sont dépourvus de droits sociaux (accès très limité au marché de I'emploi, impossibilité d'acquérir et de transmettre des biens immobiliers à l'extérieur des camps, absence de couverture sociale ${ }^{3}$ ) et ils font les frais des évolutions économiques du Liban depuis la fin de la guerre civile (1990) qui a conduit à la précarisation d'une frange de la population dont ils font partie (Doraï, 2004). Tout ce qui pourrait faciliter leur installation pérenne est ainsi écarté au prétexte

1 Anthropologue, chargé de recherche IRD, URMIS (CNRS UMR 8245 - IRD UMR 205 Universités Nice Sophia Antipolis et Paris Diderot), Case 7027, 75205 Paris cedex 13 ; nicolas.puig@ird.fr

2 Le matériau analysé résulte d'enquêtes conduites au Liban durant mon accueil au Centre d'Étude pour le Monde Arabe Moderne de I'Université Saint Joseph (CEMAM) dans le cadre d'une convention de recherche avec I'URMIS. Je remercie le directeur Christophe Varin et Liliane Kfoury, ma partenaire scientifique de I'Unité Interdisciplinaire Mémoire du CEMAM. Ce texte est une version modifiée et actualisée à la faveur d'une mission en Grèce de I'article : Puig Nicolas (2013) Lost in Transition: Ordeals of Passage of Palestinian Refugees in Lebanon, Mediterranean Politics, 18 (3), pp. 394-410.

3 Voir dans ce numéro le texte d'Assaf Dahdah. 
que la "libanisation ${ }^{4}$ des réfugiés risquerait de mettre en péril les fragiles équilibres communautaires nationaux.

Plus des trois quarts des Palestiniens (environ 250000 personnes) résident encore dans des camps disséminés dans les grandes villes du pays dont les entrées sont gardées par des barrages de I'armée libanaise. À l'exception des camps de Beyrouth, de ceux de Badawi au Nord et d'al-Buss au Sud, il en est ainsi à Tripoli, à Saïda et à Tyr. La relégation spatiale et statutaire des réfugiés s'accompagne d'une méfiance de la part de la population "locale " : les différents épisodes de la guerre du Liban ont laissé des traces quasi indélébiles. Aujourd'hui les possibilités d'émigrer à l'étranger sont extrêmement rares et dépendent d'un mariage avec un expatrié (la plupart du temps un membre de la parentèle ayant précédemment migré) ou de la poursuite d'études supérieures qui concernent très peu de personnes. Dans ce contexte, la migration clandestine devient un recours pour beaucoup de jeunes hommes et se présente bien souvent comme un adjuvant face aux difficultés et aux contraintes qu'ils rencontrent au Liban.

Cette position de relégation ne va pas sans nombre d'entraves qui affectent les mobilités des réfugiés et rendent les passages des frontières problématiques. De fait, les mobilités tant à une échelle nationale qu'aux échelles internationales impliquent des transitions entre des mondes distincts qui nécessitent soumission aux procédures formelles de classement des personnes aux lieux de contrôle (barrages de l'armée à l'entrée des camps, frontières entre les États) et adaptation aux univers de l'autre à l'occasion d'interactions intervenant dans les lieux de migration.

Car ces transitions ne concernent pas seulement des franchissements spatiaux et les frontières ne s'envisagent pas uniquement sous leur dimension territoriale : sociales et culturelles, elles signalent des écarts entre des modes de vie, des habitudes collectives et des apparences qui différencient au sein de la ville libanaise comme dans le pays lointain.

Toutes ces frontières comprennent une dimension relationnelle ; quelle que soit leur nature, elles impliquent un contact avec un autre détenteur d'une légitimité : celle conférée par les autorités territoriales ou celle offerte par une citadinité et une citoyenneté dans les villes fréquentées. Le contact avec ces différents représentants des ordres politiques et urbains s'établit la plupart du temps en défaveur des réfugiés/migrants pour lesquels le moment crucial du passage est en conséquence un moment de tension. Aussi les habitants des camps au Liban insistent-ils sur la nécessité de maîtriser les passages de frontières. Ils tentent de développer au mieux leur capacité de franchissement, et élargissent leur compétence communicationnelle, par exemple en apprenant des langues étrangères. Ces ressources représentent alors un élément majeur de leur panoplie existentielle constituée par l'ensemble des moyens à leur disposition pour avoir prise sur le cours de leur existence.

4 Le terme utilisé est tawtîn. Il désigne le fait d'octroyer la nationalité libanaise, or les réfugiés ne la demandent pas, mais réclament avec insistance des droits sociaux et un assouplissement des procédures sécuritaires qui les affectent. 
Autant l'observation des mobilités que les récits énoncés par les réfugiés palestiniens à leur propos soulignent la centralité des situations de passage de frontières dans le quotidien. Aussi est-il nécessaire d'accorder à la question des franchissements l'importance qui est la sienne dans l'ordinaire des circulations et d'accompagner les réfugiés dans leurs parcours. Ce sont ces " espacestemps " du passage qui nous retiendront dans cet article en nous intéressant particulièrement à trois formes de frontières ${ }^{5}$.

La première se trouve mise en évidence par une crise qui a provoqué une multiplication des mobilités aux échelles nationales et internationales. En 2007, à la suite de combats entre l'armée libanaise et un groupe jihadiste (fath al-islam), qui avait investi le camp de réfugiés palestiniens de Nahr al-Bared situé au nord du Liban, la partie originelle d'un kilomètre carré fondée en 1949 fut totalement détruite et les extensions, appelées " nouveau camp ", partiellement endommagées. Les habitants qui avaient été déplacés lors du conflit ne sont rentrés que progressivement à Nahr al-Bared à partir du mois d'octobre 2008. Encore en 2011, une partie importante des réfugiés n'avait pas pu rejoindre son lieu de vie, attendant dans des hébergements de fortune la livraison des derniers programmes de reconstruction mis en œuvre par I'UNRWA.

Depuis cet évènement, les réfugiés ont l'obligation de présenter une autorisation des services de l'armée pour entrer dans Nahr al-Bared par I'un des cinq barrages qui séparent le camp des espaces libanais environnants. Le premier type de frontière isole donc le camp de la ville et des environnements libanais II constitue un lieu révélateur de l'assignation identitaire où les réfugiés doivent justifier leur passage et où ils sont susceptibles de subir regards et remarques dépréciatives des soldats.

Les passages deviennent des traversées quand ils s'inscrivent dans des trajectoires transnationales. Les traversées représentent la deuxième forme de frontière. Elles désignent la migration vers la Grèce par une route clandestine, terrestre jusqu'en Turquie, puis maritime pour la dernière étape. De façon générale, les jeunes migrants qui l'empruntent espèrent ensuite rejoindre l'Europe du Nord. Cette route constitue le deuxième axe migratoire en Méditerranée avec près de 25000 passages illégaux de la frontière grecque ; le premier axe étant la route centrale méditerranéenne avec 40000 passages, selon les données 2013 de l'agence Frontex 6 . On relève que depuis une quinzaine d'années, avec des pics de départ durant la guerre deTrente-trois Jours en 2006 et lors de la bataille de Nahr al-Bared, de nombreux jeunes réfugiés palestiniens sont ainsi partis pour Athènes en passant par la Syrie et la Turquie.

\footnotetext{
5 La description de ces passages constitue un chapitre spécifique d'un travail ethnographique portant sur les mondes palestiniens au Liban. Les données nécessaires à l'écriture de ce chapitre furent recueillies entre août 2008 et juillet 2011 lors de plusieurs séjours dans le camp de Nahr al-Bared. Elles sont complétées par quelques entretiens menés à I'association de la communauté palestinienne en Grèce et des rencontres avec plusieurs jeunes Palestiniens résidant à Athènes.

6 URL : http://frontex.europa.eu/trends-and-routes/migratory-routes-map. Les passages des Palestiniens numériquement peu nombreux ne sont pas comptabilisés ; les Syriens représentent actuellement la première nationalité à entrer illégalement en Grèce avec 12727 passages répertoriés pour l'année 2013. À propos de Frontex, voir le texte d'Olivier Clochard dans ce même numéro.
} 
Quand ils parviennent enfin à prendre pied dans la capitale grecque, les migrants doivent s'adapter à la grande ville et se faire une place dans son organisation économique et sociale. Une troisième forme de frontière concerne alors les différences linguistiques et culturelles auxquelles les arrivants sont confrontés et elle nécessite de faire face aux divers effets des interactions sociales, notamment quand il s'agit de négocier leur présence en Grèce.

Les entretiens menés à Nahr al-Bared avec les personnes qui ont pris la décision de rentrer au camp après un séjour difficile en Grèce font émerger des récits d'échec témoignant du rejet des migrants-étrangers par la ville d'Athènes. Les jeunes reconsidèrent alors les normes d'une vie acceptable à la lumière de leur échec migratoire. La situation est presque pire pour la plupart de ceux restés à Athènes qui se trouvent pris au piège de la ville sans pouvoir la quitter, ni être en mesure de s'y stabiliser dans des conditions correctes.

Les trois frontières décrites ne sont donc pas de même nature, néanmoins leur franchissement met en jeu des processus individuels et collectifs d'identification et de différenciation qui déterminent une présence au monde spécifique. Chacune de ces frontières implique des définitions plus ou moins explicites des personnes pour instaurer des procédures de distinction et/ou attribuer des caractères particuliers aux réfugiés et aux migrants. Notre propos dans cet article visera donc à mettre en lumière certains des enjeux que recèlent ces " espaces-temps " particuliers du classement et de la négociation, des découvertes et des rétractations, des ouvertures et des enclavements en suivant le point de vue des réfugiés palestiniens.

\section{Premier passage : barrages militaires et assignations identitaires}

En 2006, Abdallah Wahbé, I'un des musiciens de I'orchestre Al-Karmel ${ }^{7}$ écrit et enregistre une chanson, dans I'un des homes studios du camp de Nahr al-Bared, intitulée "Hawajiz" (checkpoints). Les paroles critiquent les barrages militaires qui entourent le camp. Le musicien insiste sur l'humiliation quotidienne, à tout le moins sur le profond inconfort, que représentent ces passages obligés pour entrer ou sortir du camp. Six ans plus tard, en 2012, la situation a empiré pour les Palestiniens : non seulement les checkpoints sont toujours présents, mais les contrôles ont été renforcés depuis le conflit de 2007.

\section{La situation dans le camp de Nahr al-Bared}

En effet, à la suite des événements de 2007, un permis a été institué sans lequel les habitants palestiniens ne peuvent entrer dans le camp. En juin 2012, en opposition à l'ordre militaire instauré sur le camp, les réfugiés manifestent pacifiquement durant douze jours. Le mouvement de contestation débute après la mort d'un jeune atteint par la balle d'un militaire libanais. II protestait avec d'autres contre la confiscation d'un scooter d'un habitant du camp, sous prétexte que le conducteur $n^{\prime}$ avait pas des papiers en règle. La protestation

7 Cet orchestre est affilié au Front Populaire de Libération de la Palestine et il est spécialisé dans la musique nationaliste et " patrimoniale " palestinienne. 
s'intensifie durant les funérailles, car le jeune homme a dû être enterré dans une fosse commune. L'armée avait en effet interdit l'accès au nouveau cimetière considéré comme faisant partie d'une zone militaire. Le manque de place dans le vieux camp ne laissa d'autre choix aux habitants que d'enterrer le défunt dans l'ancienne fosse commune. Le mouvement de protestation qui s'ensuit provoque la mort d'un autre homme et fait plusieurs blessés. En réponse à ces violences, les habitants organisent un sit-in dans le centre du camp pour faire valoir leurs droits.

Ces évènements dramatiques soulignent l'exaspération et le ressentiment des habitants de Nahr al-Bared envers les procédures de sécurité auxquelles ils sont collectivement soumis alors même qu'ils sont les premières victimes de la bataille ayant conduit à la destruction de leur camp dans laquelle ils ont perdu leur habitation et leurs effets personnels. La dénonciation du comportement de l'armée qui a conduit au décès de deux habitants s'est concentrée sur l'ordre militaire qui régit le camp. La première revendication portait sur l'élément le plus contraignant de cet ordre : I'abolition des permis d'entrée dans le camp. Finalement, l'armée accepta de céder à cette exigence, mais les habitants doivent encore présenter leur carte de réfugiés. Les checkpoints demeurent et les voitures et bagages font encore l'objet de fouille au cas par cas.

En aval du passage, le mécanisme de l'assignation identitaire découlant de l'assujettissement à l'ordre militaire concentre le ressentiment des réfugiés, comme en témoigne ici Saleh, un habitant de Nahr al-Bared qui a récemment tenté de passer en Grèce ${ }^{8}$ :

"La réalité quotidienne prend toujours le dessus. C'est-à-dire, imagine-toi par exemple toi : ici tu dois toujours avoir sur toi ta carte et ton laissez-passer, et tu dois déclarer chaque matin moi je suis un réfugié palestinien; tu circules au Liban et tu leur dis pour te présenter: "moi je suis un réfugié palestinien". Là-bas, tu voyages dans toute l'Europe personne ne te pose une question. Moi je ne fais pas l'éloge de l'Europe, mais il y a une réalité qui existe ! [...]".

Cette situation a considérablement limité la fluidité des passages et établi une nouvelle configuration du contrôle d'où a émergé un moment social spécifique. Elle a ouvert un nouveau chapitre dans le dense livre des expériences du passage des réfugiés palestiniens que je propose de détailler.

\section{Le checkpoint comme lieu d'une rencontre asymétrique}

Les observations des interactions entre les soldats et les habitants du camp, ainsi que les discours recueillis à ce propos permettent de documenter les passages des barrages de l'armée, au moins partiellement, car l'enquête se révèle délicate. Les habitants doivent emprunter I'un des cinq checkpoints qui gouvernent l'entrée dans le camp. Ils se déterminent en fonction de la proximité du point de contrôle, de la densité du trafic, mais aussi en fonction des critères plus subjectifs : la relation avec les soldats est supposée plus facile et détendue

8 Les traductions de I'arabe vers le français sont de Liliane Kfoury, que je remercie chaleureusement, révisées par l'auteur. Je remercie également Wissam Ridha de Nahr al-Bared, passionné du "voyage ", qui m'a accompagné durant les enquêtes sur les migrations. 
dans le petit poste nommé al-Bayadé situé sur une route secondaire conduisant au camp, que dans ceux ou le trafic est plus important, à l'instar des barrages de Mouhamara ou de al-Abdé situés sur la route reliant Tripoli à la Syrie.

C'est par al-Bayadé que l'on me fit passer un jour alors qu'invité à un mariage, j'avais omis de faire renouveler mon autorisation périmée tandis que les bureaux de l'armée à Tripoli étaient fermés. Hassan, une connaissance du camp, se trouvait justement dans cette ville et se proposa pour m'accompagner et tenter le passage. II fit le pari que le militaire penserait moins à regarder la date du permis qu'à se concentrer sur la vérification de mon identité. Sur la route, Hassan ne manifesta aucun signe d'inquiétude ${ }^{9}$. Nous passâmes néanmoins quelques minutes tendues quand le soldat s'arrêta sur les différences de graphie arabe entre le permis d'entrée et mon passeport. Néanmoins, il ne se rendit pas compte que mon permis avait expiré et nous entrâmes dans le camp sans encombre. La nouvelle de notre passage se propagea rapidement parmi la noce, et les nombreux commentaires enthousiastes que suscita ce modeste succès résonnèrent comme une sorte de petite victoire symbolique témoignant de l'importance collectivement accordée à l'ordre militaire mis en place aux checkpoints.

L'une des notions mobilisées de façon récurrente pour donner un sens à la situation de passage du barrage militaire est celle d'étrangeté ${ }^{10}$ (gharîb). Évoquer une étrangeté pour décrire les diverses interactions au barrage, comme à la ville, renvoie à une incertitude quant à ce qui est partagé avec autrui et au doute concernant la possibilité d'un échange pacifique et égalitaire. Ainsi, les passages des checkpoints qui prennent place au long des routines quotidiennes ne sont jamais anodins. Chaque signe, même la plus discrète manifestation de mépris de la part d'un soldat, est perçu et interprété.

Cette étrangeté provoque une gestuelle de l'étonnement ou de l'impuissance : contraction du visage, haussement de sourcils, mouvement des mains, position corporelle de retrait. Le passage des barrages de l'armée annihile le désir de normalité des réfugiés et cristallise les tensions d'une rencontre asymétrique où I'on est soumis à une assignation identitaire sur laquelle on ne dispose que de peu de prise. Cela entraîne une adaptation spécifique dans l'interaction, graduée selon les cas de l'interrogation du regard, de la démonstration de la surprise, à l'expression (risquée) de son mécontentement, en passant par l'ironie dissimulée. Puis, dans le huis clos de l'entre-soi, une fois regagné sa place dans la voiture ou le minibus, les commentaires fusent comme autant de

9 À l'inverse des shababs (jeunes) du camp, Hassan ressent une certaine proximité avec les militaires libanais du fait du mariage de sa sœur avec un soldat. De plus, il a des origines libanaises : son grand-père libanais avait dû s'enfuir en Palestine pour échapper à un mandat d'arrêt. II revint au Liban en 1948 avec les réfugiés palestiniens devenant depuis lors l'un d'entre eux.

$10 \mathrm{~J}$ 'ai eu l'occasion de décrire à propos des pratiques urbaines des réfugiés palestiniens au Liban, I'emploi de ce terme dans une opposition avec celui d'intimité (Puig, 2012 : 237-239). L'intimité souligne une familiarité ou une privauté. Les deux notions, intime et étrange, sont proches de l'expérience : I'intime sanctionne une forme d'entresoi instituée par différents processus comme la circulation des rumeurs par exemple, ou encore les situations d'énonciation qui créent un espace discursif de la proximité. II qualifie ensuite des pratiques spécifiques qui sont d'ordre privatif et peuvent marquer une distance prise avec l'ambiance sociale et morale du camp (boire de l'alcool, flirter, etc.), en investissant des espaces urbains extérieurs comme un chez soi alternatif. 
petits actes réparateurs destinés à maintenir la dignité. Le registre de l'étrange mobilisé dans ces courts dialogues, " ce soldat était bizarre ", témoigne d'une façon de mettre à distance la stigmatisation. Car le passage du barrage expose les réfugiés à des signes tangibles d'irrespect exprimés dans les comportements des soldats : les regards, les mots et la façon de les dire, l'attitude corporelle rendent alors perceptible une certaine antipathie. En aval, la gêne du barrage provoque des réponses militantes, mais aussi culturelles. Un exemple récent qui a marqué les esprits dans le camp concerne la chanson d'un rappeur de Nahr al-Bared, DJ Tamarrud (DJ Rebelle) dont le but est de restaurer la dignité des réfugiés: "Si tu as une carte d'identité bleue, relève la tête " chante-t-il. La vision d'un vieil homme malmené au barrage qui lui semblait honteux de sa situation de réfugié lui a inspiré ces paroles ${ }^{11}$.

La dignité du passage dépend de l'attitude des soldats plus ou moins conciliants, aimables ou hostiles. On doit s'en remettre alors à la présence chez ces derniers d'un sentiment d'humanité partagé à laquelle renvoie la formule récurrente chez les individus subissant une stigmatisation : "On est des hommes comme les autres, on est des hommes comme vous ". Une rhétorique de I'humain, car elle transite donc par le langage, est ainsi régulièrement mobilisée pour faire valoir une revendication de traitement digne. Elle est opposée par les réfugiés à I'assignation identitaire inhérente au barrage, au-delà du cas présent, cette rhétorique est mobilisée de façon universelle par les personnes qui subissent une situation de stigmatisation.

Les gestes de sollicitude des soldats sont perçus au prisme de cette grille de lecture qui oppose humain et inhumain. Au passage du barrage d'al-Bayadé pour entrer dans le camp, Sayyid s'avisa que son portefeuille contenant ses papiers et, surtout, son permis d'entrée dans le camp, avait glissé de sa poche. Tandis qu'il commençait nerveusement à les chercher dans la voiture, le militaire conciliant lui dit " $c^{\prime}$ est peut-être tombé sous le siège "; il lui enjoint de prendre son temps, en lui glissant un " yallah bassita " (" allez, ce n'est rien ") apaisant. Une fois les papiers retrouvés ${ }^{12}$, Sayyid commenta la situation en insistant sur " I'humanité " (insânîyé) de ce militaire. À l'inverse, l'emprise sécuritaire sur le camp et son lot d'humiliations suscite nombre de discours sur le caractère " inhumain " du sort réservé aux réfugiés. Ce constat est partagé par l'ensemble des réfugiés au Liban : Perdigon (2008) note par exemple l'omniprésence du vocabulaire de l'inhumain dans les conversations des résidents du groupement palestinien de Jal ad-Dib àTyr.

Le passage des checkpoints actualise le statut des réfugiés et souligne leur situation de " marginaux " au Liban. Face à une marginalité ressentie autant comme un empêchement que comme une indignité, nombreux sont ceux qui cherchent à échapper à cette situation et à accéder à une autre vie en quittant le pays, la plupart du temps de façon clandestine. Ainsi, la plupart des jeunes hommes n'ont d'autre choix que de se lancer dans un périlleux voyage vers I'Europe qui implique de transiter par la Grèce, premier pays européen accessible.

11 URL : http://www.youtube.com/watch?v=61 macFunihU

12 Si tel n'avait pas été le cas, Sayyid ne serait pas entré dans le camp et n'aurait pu regagner son domicile. 


\section{Deuxième passage : traversées vers la Grèce}

Le voyage vers la Grèce est un autre type de passage que celui envisagé ci-dessus. Depuis les premières années du XXle siècle, de nombreux jeunes palestiniens de Nahr al-Bared se sont lancés dans une traversée clandestine vers la Grèce. Les shababs (il s'agit exclusivement de jeunes hommes) espèrent rallier le nord de l'Europe, en particulier l'Allemagne et la Scandinavie où se trouve une importante diaspora palestinienne et où chaque habitant de Nahr al-Bared a au moins un membre de sa famille susceptible d'accueillir le nouveau migrant (Doraï, 2004). Mais la plupart du temps, les jeunes ne parviennent pas à dépasser Athènes et restent dans la ville entre six mois et un an, parfois plus. Bloqués dans la capitale grecque et dans l'incapacité de poursuivre la route, certains décident de retourner au camp muni d'un laissez-passer délivré par I'ambassade du Liban en Grèce.

Durant leur voyage vers la Grèce, les shababs connaissent une situation d'entre deux, de liminalité " dans laquelle les individus pris un temps entre deux états (comme migrants sur la route et comme immigrants, l'état auquel ils aspirent) font l'expérience de la situation ambivalente caractéristique des gens du seuil ${ }^{13}$ (Streiff-Fénart et Segatti, $\left.2011: 26\right)$.

\section{" Arches de Noé " de la précarité}

Il était possible de se rendre en Turquie depuis la Syrie jusqu'à ce que l'intensification des combats au début de l'année 2012 rende le transit par ce dernier pays trop dangereux. À I'heure actuelle, si de très nombreux Syriens franchissent la frontière avec la Turquie pour rallier l'Europe, il est désormais nécessaire d'obtenir un visa touristique pour se rendre à Istanbul en avion depuis le Liban et ensuite traverser vers la Grèce.

La suite de la route demeure la même : on s'embarque, généralement des environs d'Izmir, en direction de l'une des îles grecques toutes proches. Le coût d'un tel trajet est très variable et dépend notamment du temps passé en Turquie en attendant d'embarquer. Aussi le prix oscille-t-il entre 1000 et 5000 dollars. Au-delà du prix, le voyage n'est pas sans danger et deux jeunes originaires de Nahr al-Bared ont péri dans le naufrage du bateau qui devait les amener en Grèce au milieu des années 2000.

La description de ces voyages clandestins par les réfugiés-migrants superpose des images de paysages menaçants et des ambiances nocturnes : montagnes entre la Syrie et la Turquie ou bras de mer séparant les côtes des îles grecques. Ces paysages sont aussi peuplés de migrants de toutes origines, de passeurs, de policiers et de gardes-frontières. Les Palestiniens font alors l'expérience d'une société temporaire composée d'exclus des mobilités légitimes qui se déplacent clandestinement de nuit et voyagent sur les " arches de Noé " précaires qui sillonnent les différentes parties de la Méditerranée. Najdé en donne une description saisissante :

13 " [A situation] in which individuals caught for a time between two states (as migrant on the road and as immigrant, the state to which they aspire) experience the typically ambiguous situations characteristic of threshold people " (traduit par I'auteur). 
" On est arrivé à l'endroit d'où on devait partir pour la Grèce, une langue de terre d'une centaine de mètres descendant en pente douce vers la mer, au milieu d'une très grande montagne divisée par une fente, en deux parties élevées. Quand on est arrivé à la mer, j'ai eu la chair de poule en voyant une centaine de gens de tous âges et de toutes origines. Des enfants d'un mois et de deux, des filles, des jeunes femmes, des femmes, des vieux, des jeunes, des Égyptiens, des Palestiniens, des Somaliens, des Bangladais. On était deux groupes à partir en Grèce. En attendant, on s'est mis à discuter ensemble.

Le passeur, impatient, a contacté le bateau qui était en retard. Le grand bateau avançait dans notre direction. II a accosté à une distance de 100 à 150 mètres de la plage. On a installé un ponton gonflable pour traverser de la plage jusqu'au navire. Avec quatre autres jeunes hommes et trois Égyptiens, nous avons aidé les petits et les femmes à traverser $"$.

Dans ce contexte la présence de migrants illégaux de diverses origines produit des moments de sociabilités où l'on use de stéréotypes nationaux lors des interactions. Najdé en donne un exemple en décrivant une situation tendue :

"Par hasard, le Kurde est arrivé avec sa femme, son frère, et ses enfants. II s'était débarrassé de son canif lorsqu'on est arrivé sur l'île pour qu'on ne l'arrête pas. Quant à moi, j'avais pour me soutenir, Ibrahim, Firas et Adham. Ils prenaient tous mon parti. Le Kurde est arrivé près de nous, je lui ai enfoncé la branche d'arbre dans le cou et je lui ai dit: "tu pensais menacer qui avec ton couteau? Tu ne sais pas qui nous sommes? Tu n'as jamais entendu parler des Palestiniens? Je jure que je peux te réduire en morceaux ici même. Ce n'est pas cela que tu voulais, me massacrer ?". Il a répondu qu'il était désolé et que son intention était d'arriver à destination et qu'il n'a pas voulu... Je lui ai dit: "Écoute, c'est la première et la dernière fois ". Son frère et sa femme ont commencé à me supplier et me dire qu'ils sont venus ici pour immigrer... Ibrahim est intervenu, il m'a dit: "laisse-le ". J'ai dit: "OK ça marche" ".

\section{Des vaches et des hommes}

La description de Najdé prend des accents épiques quand il décrit la rencontre de la montagne et de la mer:

"Une plage avec d'immenses et fascinants rochers [...], de hautes vagues. On est descendu du bateau qui n'a pas été amarré sur la plage pour éviter qu'il ne s'enfonce dans le sable. On nous a fait descendre dans l'eau. J'ai soulevé ma veste sur la tête et j'ai gardé mes vêtements. J'ai avancé jusqu'à la plage, l'eau atteignait mes genoux.

On a porté les enfants un à un, on a aidé les femmes à avancer en les tenant par la main, on a tous débarqué. Sur l'île, on s'est séparé et chacun est parti de son côté. Les Somaliens sont partis d'un côté, les Bangladais de l'autre, nous, Palestiniens, avons pris un autre chemin et un immigrant clandestin comme nous, originaire de la Turquie, nous a accompagnés [...]. Vers 7 heures, 7 h 30, on a enfin atteint une route. On a escaladé des montagnes et on a descendu des montagnes, on errait... On montait sur une montagne très élevée et on redescendait en nous retrouvant en face d'une autre montagne, jusqu'au moment où on est arrivé sur l'autoroute".

Malgré le caractère dramatique de ces descriptions, Najdé conserve le sens de I'humour quand il raconte le passage de la frontière accompagné par des vaches que le passeur achemine de Syrie en Turquie dans le même " convoi " que les migrants : 
"Les vaches ont pris la fuite avec nous. En marchant, le passeur nous a recommandé de tirer la bête par la queue pour qu'elle avance plus vite. "Hurr, Hurr, Hurr, Brr, Brr, Brr. Attrapez la bête et poursuivez-la!". Et on se précipite et on chasse les vaches. On s'est arrêté quelque part pour se reposer. Au lieu de nous rassurer et de nous remonter le moral, le passeur a rendu la situation encore plus insupportable en nous racontant ses mésaventures à cet endroit: "Une fois, je passais par là avec des voyageurs clandestins et on a commencé à nous tirer dessus". On a senti qu'on était à bout de nerfs. On lui a demandé pourquoi nous raconter une histoire pareille: "Plutôt que de nous apaiser, tu nous apprends qu'on a tiré sur vous ici ? Pourquoi tu nous fais peur ?". II a répondu : "Ce n'est pas pour vous faire peur, mais je dis la vérité". Je lui ai dit: "Espérons que tout se passera bien. Que Dieu soit avec nous. Avançons" ".

Après la traversée maritime et le débarquement dans I'une des îles grecques (Samos, Lesbos, Chios ou Rhodes), le voyage s'achève immanquablement par une arrestation. À ce moment, les Palestiniens espèrent encore obtenir un traitement de faveur de la part des autorités grecques. Ils prennent soin de se démarquer des migrants économiques pour faire valoir leur légitimité à demander l'asile qui doit déboucher sur l'acquisition d'une nationalité comme en témoigne par exemple ce père de famille:

" J'ai voyagé, car je suis une personne instruite, j'ai l'ambition d'un meilleur avenir. Mais je vis au Liban [où je suis réfugié] : pourquoi je ne peux pas vivre comme les gens ici, comme n'importe quel Libanais ? [...] Alors j'ai commencé à penser à l'Europe, comme on la voit à la télé... mais tout ça c'est du vent, du mensonge. [...] Moi avant j'avais de l'argent, j'avais beaucoup d'argent, je suis parti juste pour que mon fils ait un avenir, pour qu'il ait une nationalité ".

Pourtant, les réfugiés palestiniens subissent le même traitement que les autres migrants. Baha, comme nombre de ses compatriotes, pense que l'image des Palestiniens a changé du fait que de nombreux Arabes se présentent comme Palestiniens pour bénéficier d'un meilleur accueil de la part des autorités comme de celle des citoyens grecs:

"Ils aimaient beaucoup les Palestiniens; ils aimaient beaucoup, beaucoup les Palestiniens, mais ce qui est arrivé, le peuple grec aime les Palestiniens, aime Yasser

Arafat, la police, tout le monde nous respecte, mais ce qui est arrivé ces six, sept dernières années, quand par exemple l'Algérien volait, le Maghrébin faisait des histoires à l'Égyptien, il allait tuer, tout, le Syrien qui vendait de la drogue ; quand il était arrêté, il disait qu'il était Palestinien ; le mot "Palestinien" l'aidait ".

Jihad que je rencontrais à Athènes en novembre 2013 tient exactement le même discours :

"C'est-à-dire l'Algérien vient et s'enregistre en tant que Palestinien, le Syrien venait et s'inscrivait comme Palestinien, parce que le Palestinien on ne le rapatrie pas dans son pays; ils ne peuvent pas le rapatrier en Syrie, ils ne peuvent pas le faire repartir. Ils trouvent donc qu'une pression sur le Palestinien, c'est mieux. Dans le temps, par le passé, ils nous aimaient ici, les gens ; mais ils nous ont haïs. Ils ne nous ont pas haïs en tant qu'individus, ils ont détesté les actes. Les actes, c'est-à-dire l'Algérien qui s'inscrit comme Palestinien, va voler dans un centre commercial et on dit, c'est un Palestinien". 
À la suite d'une détention administrative d'une durée variable, les migrants sont libérés avec une autorisation temporaire de séjour, ils regagnent alors Athènes où la joie d'être parvenu en Europe cède rapidement la place aux désillusions face aux difficultés à prendre place dans la ville lointaine.

\section{Troisième passage : les écueils de la ville lointaine}

Avant le départ pour la Grèce, les conversations des jeunes hommes dans le camp tournent autour du voyage et de l'intégration en Europe. La clandestinité du trajet fait l'objet de discussions serrées tandis que la connaissance de certaines affaires lointaines au travers des canaux relationnels avec les expatriés (Facebook, Skype, applications de smartphones) laisse à penser que tout n'est pas idyllique là-bas. Les échos de nuits passées dehors, de passage à tabac par la police, ou encore de racisme arrivent jusqu'au camp colportés notamment par ceux qui sont revenus de Grèce. Ces derniers ont fait le choix du retour face aux épreuves endurées à Athènes, ville dont ils ont rêvé avant d'en éprouver la dure réalité. Dans la capitale grecque, la frontière prend la forme d'un écart entre les réfugiés et les personnes rencontrées. Du fait de l'enclavement progressif du mode de vie des Palestiniens à Athènes, les rencontres avec les Grecs tendent à se limiter à quelques personnes qui chacune constitue une sorte de mise à l'épreuve : un couple exhibitionniste, un policier raciste ou encore un patron voleur, etc.

\section{Khalil : l'expérience de l'impudeur}

La première rencontre avec Khalil a lieu dans son magasin, juste devant le camp, sur l'autoroute qui relie Tripoli à la frontière syrienne. Elle se déroule lors d'une journée de déluge comme en connaît le Liban de temps à autre durant I'hiver. Mais, à l'inverse de ceux qui se plaignent des rudesses climatiques, Khalil se félicite du mauvais temps. Car la foudre provoque des perturbations dans les circuits électriques, qui font griller les télés et il est justement spécialiste de leur réparation. De retour à Nahr al-Bared après un séjour d'une année en Grèce, Khalil vient, en effet, de reprendre son ancienne activité.

II affiche une grande modestie dans ses objectifs de vie et insiste sur ses ambitions désormais extrêmement limitées : il se contente d'apporter à sa famille de quoi se nourrir, même s'il reste fier de son métier et de son aptitude à le faire avec compétence. II en retire une évidente satisfaction qui ressort de sa description de ses relations avec ses clients souvent libanais qui le préfère aux " fils du pays " plus chers et moins adroits.

À plusieurs reprises, il me propose de passer chez lui, il souhaite me montrer comment il vit et insiste à décrire les fondations de son quotidien qui rassemble désormais toute son énergie : la maison, la nourriture, les enfants, les routines du magasin de réparation de télé. II me confie n'avoir plus vraiment de but, juste se satisfaire de ce qu'il a ; il ne s'en sort pas si mal avec son magasin, la pêche le dimanche, une sortie familiale de temps à autre à Tripoli. Cette organisation du quotidien le rassure, elle "répare " en quelque sorte la vie abimée par la 
migration en offrant une certaine "sécurité ontologique "(Giddens, 1991) ${ }^{14}$.

À l'instar de Khalil, les réfugiés-migrants en revenant chez eux entrent à nouveau dans la " temporalité du camp "15 (Agier, $2012: 35)$. Cette rentrée se manifeste par un acte fondateur qui signale que le retour est durable, sinon définitif. Ainsi la plupart des migrants " ouvrent une maison ", selon l'expression en vigueur, c'est-à-dire qu'ils fondent une famille et s'installent dans leur propre appartement quand ils en ont la possibilité.

Khalil a décidé de quitter la Grèce du fait de ses difficultés à trouver une place dans un pays européen. Il évoque à ce propos des raisons de moralité religieuse. Peu importe que cette explication masque l'échec à prendre pied véritablement en Europe ou qu'elle atteste bien de la difficulté à s'ajuster à une différence indépassable, elle est un élément central de son récit sur son expérience grecque :

" Je suis parti, on est allé en Turquie. Moi avant j'avais de l'argent, j'avais beaucoup d'argent [...] quand je suis allé, j'ai vu, j'ai beaucoup changé, parce que je suis musulman. [...] Mais le peuple grec est très bien, ils aiment beaucoup les Palestiniens. Dès qu'ils savaient que j'étais Palestinien, il me disait "fuck America, fuck Israël" ".

Khalil est ainsi le témoin d'une scène dans le métro d'Athènes qui le met mal à I'aise. À la vue de tout le monde, un jeune couple, un garçon et une fille flirtent sans inhibition, le garçon remontant la jupe de sa compagne et lui caressant les fesses. Cette scène, qui l'indigne autant qu'elle accentue son mal-être à Athènes, le décide à retourner au Liban bien qu'il soit, selon ses dires, l'un des rares migrants palestiniens de l'histoire récente à réussir son intégration économique. En tant qu'électricien qualifié, il s'est rendu indispensable au bon fonctionnement d'un magasin d'électronique :

"Alors moi j'étais assis [dans le métro] et je pensais comment je vais amener ma femme et ma fille voir... en islam, ça est un grand, grand problème. À ce moment, je me suis dit: "rentre". Ce moment m'a laissé réfléchir ; c'est fini je retourne [...]. Ça m'a fait revenir. Moi je vivais content, mais ça, ça m'a fait revenir. C'était trop. Il y avait trop de libertés [...] ".

14 Une définition générale de la sécurité ontologique : " La notion de sécurité ontologique est étroitement liée au caractère implicite de la conscience pratique - ou en termes phénoménologiques, aux ajustements requis par l'attitude naturelle dans la vie quotidienne " (op.cit. : 36, traduit par I'auteur : "The notion of ontological security ties in closely to the tacit character of practical consciousness - or, in phenomenological terms, to the "bracketings" presumed by the "natural attitude" in every day life "). $\mathrm{L}^{\prime}$ « attitude naturelle " implique la conviction que le monde et ses objets sont tels qu'ils nous apparaissent (Schütz, 1987 : 127) ; elle entraîne une suspension du doute, dans le cas des Palestiniens de retour au camp, cette suspension est permise par l'adoption d'une existence resserrée autour des espaces familiers et de l'instauration d'un mode de vie routinier. Cet investissement dans le quotidien n'est pas sans rappeler également les réflexions de Veena Das qui décrit la descente dans le quotidien de personnes ayant été confrontées à des violences extrêmes (cité par Perdigon, 2010 : 974).

15 Pour définir cette figure de la temporalité du camp, Agier écrit : «De manière très pragmatique, l'exilé vit, survit, fait des rencontres, organise son existence. Un temps réel s'installe quand le présent s'allonge. Mais il n'est encore rien d'autre qu'un présent qui dure. Dans l'espace réel des camps, quel sens est associé à la possibilité de décrire le lieu lui-même dans la durée ? Le camp est la manifestation d'un présent immédiat parce qu'il exclut le passé et l'avenir » (2012: 35-36). Si les modes de vie adoptés par les réfugiés de retour de Grèce se cristallisent bien autour de la quotidienneté, l'idée d'un présent qui dure doit être nuancée dans le cas des camps palestiniens, car ils ont généré leur propre historicité en plus de soixante ans d'existence. 
La réaction de Khalil renvoie à l'une des visions des villes européennes ayant cours dans le camp. Celle-ci inspire la peur d'une dissolution morale et de la perte des valeurs. On craint pour les enfants que l'on imagine démunis face à ses tentations, notamment celle de la drogue. Par contraste le camp est perçu comme un lieu où les valeurs collectives sont encore présentes. À tel point qu'il n'est pas rare que des pères vivant à l'étranger y envoient leurs enfants durant les vacances d'été, quand la situation sécuritaire le permet, dans l'espoir qu'ils acquièrent certaines des valeurs de la communauté.

La crainte de la ville est liée à ce qu'elle pourrait donner à voir, dans ses espaces publics, comme le métro exemple où spectateur malgré soi on reste prisonnier de scènes impudiques. C'est ainsi par la vue que Khalil, qui revient deux fois sur la scène à laquelle il a assistée, construit d'abord son rejet de l'Europe ${ }^{16}$.

Nizar quant à lui a fait d'autres rencontres dans lesquelles il a tenté en vain de faire valoir son point de vue.

\section{Nizar : l'expérience du racisme}

Nizar a une expérience de la Grèce un peu différente. Il y a passé sept années durant lesquelles il a joué un rôle d'intermédiaire pour ses compatriotes originaires de Nahr al-Bared et des camps de réfugiés du Liban. II se plaît à dire que son appartement était l'ambassade de Nahr al-Bared à Athènes. Sa connaissance correcte de la langue grecque et une relative intégration lui permirent d'occuper cette position auprès de ses amis et connaissances débarquant dans la cité.

En témoignage de son intégration, il conserve dans ses maigres archives personnelles les photos de sa participation aux manifestations de l'été 2008. L'une d'entre elles fait sa fierté : il pose avec un drapeau palestinien entouré de jeunes Grecs et de compatriotes palestiniens. En dépit de sa longue expérience en Grèce, Nizar explique s'être fait duper par son employeur qui ne lui a jamais payé la somme de 2000 euros qu'il restait lui devoir, cela alors qu'il a travaillé pour lui durant des années. Le fait qu'il soit dans l'incapacité de se défendre a rendu l'expérience encore plus désagréable. Il lui était bien entendu impossible de porter plainte à la police, car ses demandes de renouvellement de son permis de résidence étaient systématiquement rejetées. De plus, ses rencontres avec la police devenaient difficiles, et il devait sans cesse justifier de sa présence en Grèce, selon ses souvenirs :

"Le peuple grec est un peuple raciste, franchement un peuple raciste et moi je les ai fréquentés sept ans ; ah oui, un peuple très, très raciste ; il ne t'aime que par intérêt; s'il veut quelque chose de toi, c'est par intérêt.

[...] Un policier m'a arrêté, il m'a dit: "Qu'est-ce que tu fais ici ?"... J'étais près de la maison; je lui ai dit: "Je rentre chez moi "... II m'a traité de malaka, c'est-à-dire malaka est une insulte... II me dit: "Espèce de... Qu'est-ce que tu fous en Grèce" ; je lui ai dit:

$16 \mathrm{Ce}$ qui est symptomatique d'une urbanité spécifique qui privilégie la vue sur l'ouïe qui caractérise le régime sensoriel de la grande ville comme le note Simmel au tournant du XXe siècle (1991 : 230). II insiste sur le caractère énigmatique bien plus prononcé de I'homme qui se présente exclusivement à la vue et évoque à ce sujet un sentiment d'isolement et de désorientation qui frappe la personne privée de la compréhension orale. 
"Ah, tu veux savoir ce que je fais en Grèce ?". II m'a dit : "Oui "; je lui ai montré mon appartement [situé juste au-dessus]. II m'a redit: "Qu'est-ce que tu fais ici en Grèce ?".

"Je vis ici ". "D'où es-tu, tu vis ici ?". "Je suis Palestinien ". II m'a dit: "Pourquoi tu ne vas pas en Palestine? ". Je lui ai répondu : "Comment je vais aller en Palestine ?". II m'a dit: "Comme tu es venu, tu retournes en Palestine". Il pense que... même les policiers

ils ne savent rien; il ne connait pas la Palestine; alors il me demande pourquoi je ne vais pas en Palestine. Je dois lui dire que là-bas il y a des problèmes en Palestine... des problèmes, des tirs et nous avons fui la Palestine. Alors il me dit: "Bon, va en Bulgarie, va en Roumanie, va en Italie "; c'est ce qu'il me disait. Alors moi parce qu'il me disait ces trucs, je lui ai dit: "Je vais te dire une chose : apprends-moi comment aller en Roumanie et je te donne de l'argent [...] moi, je veux sortir de ce pays, je ne suis pas content dans ton pays [...] donne-moi de l'argent et envoie-moi en Italie ". Là, il est devenu fou, comment ça ! Je lui donne de l'argent pour partir en Italie... Je lui ai dit: "Mais c'est toi qui veux que je parte, je te dis donne-moi de l'argent, mais fais-moi sortir". Alors il m'a dit: "Va-t'en d'ici, malaka...".

En fait, il a appelé ses collègues et il leur a dit: "Il y a un Palestinien qui m'agresse ! ". Lui, ce policier, il leur dit: "Il y a un Palestinien qui m'agresse, il fonce sur moi". Alors six motos sont arrivées. Il y avait deux gars sur chacune d'entre d'elles, et c'était l'été, je portais un short et des manches courtes. Quand ils sont passés ils se sont mis à rire, sur qui ? Sur moi et sur lui. Ils lui disaient: "C'est lui le Palestinien qui t'agresse ?". Moi je suis maigre... Ils lui répétaient: "C'est ce gars-là qui t'agresse ?" [...]".

Comme beaucoup d'autres, Nizar est finalement retourné dans le camp accompagné de sa femme japonaise et l'enfant de cette dernière, issu d'un premier mariage. II avait fait sa connaissance alors qu'elle voyageait en Europe sur le même bateau qui le ramenait en Grèce après une tentative avortée de passer en Italie. Il est à présent au Liban dans I'attente du document de voyage qui lui permettrait de partir vivre au Japon avec son épouse et dont les services de la sécurité générale diffèrent indéfiniment la remise.

Les migrants une fois rentrés au camp disent l'impossibilité de vivre décemment à Athènes. Ceux qui résident encore dans la ville font le même constat et sont dans la recherche d'une issue honorable à la situation de blocage qu'ils vivent $^{17}$.

Après nombre de tentatives de trouver un emploi régulier, ils survivent à la faveur de petits boulots journaliers et arrivent à la conclusion qu'ils n'ont $d^{\prime}$ autres choix que de se lancer dans les trafics illicites ou bien de revenir au Liban. Les récits décrivent la ville comme propice à de fructueuses et ludiques explorations à l'arrivée. Puis, les attentes déçues des découvertes urbaines conduisent aux temps difficiles de l'isolement social.

17 Certains ont fait le choix d'un mode de vie dans l'illégalité. Ces derniers sont plus ou moins mis au ban par les autres migrants palestiniens comme j'ai pu le constater à l'occasion du rassemblement du dimanche sur la place Monastiraki. Kamel par exemple avec qui je discutais était visiblement tenu (où se tenait de lui-même) à l'écart des autres. II m'expliquait alors qu'un retour au camp lui était impossible, car il était trop habitué à la vie en Europe : il consommait beaucoup d'alcool et il appréciait la liberté laissée à chacun. Liberté qui lui permettait de vivre en concubinage avec une Rom bulgare, ce qui ne serait pas accepté au camp. 
Najdé par exemple décrit son enthousiasme et son envie de sortir se promener malgré la fatigue du voyage qui l'a conduit en Grèce. Avec deux autres jeunes originaires du camp, ils arrivent place Omonia grâce aux indications d'un Égyptien abordé, car il avait " l'air arabe » qui les renseigne sur leur itinéraire. Puis ils retrouvent Nizar chez lui :

"On est arrivé chez Nizar, on s'est embrassé et on s'est serré dans les bras. II m'a souhaité la bienvenue et on a discuté. On s'est installé à l'intérieur. [...] Nizar a préparé du café qu'on appelle frappé là-bas, du café glacé. II m'en a offert. II m'a dit: "Tu as sommeil ?". J'ai répondu: "Je ne suis pas venu ici pour dormir, tu vas nous emmener en promenade, je veux visiter la région et voir le monde ici". II m'a dit: "On y va". [...] On s'est précipité dehors. Comme je venais d'arriver et que c'était la première journée pour moi en Grèce, j'étais content. Quel pays ! Je venais de fouler le sol de l'Europe et pour en profiter je suis allé me promener un peu partout. Je suis rentré la nuit, je me suis couché ".

Assez rapidement, l'euphorie des premiers jours cède la place au désenchantement, car les jeunes migrants comprennent que la situation précaire dans laquelle ils se trouvent en Grèce est destinée à durer. Les espaces de la vie quotidienne se rétrécissent; I'unique lieu de rencontre se trouve sur la place Monastiraki, à proximité d'un établissement d'une chaine internationale de café. Les Palestiniens en situation de précarité à Athènes, ceux des camps du Liban, les autres venus de Syrie ou encore de Gaza, échangent quelques nouvelles autour d'un ou de plusieurs cafés le dimanche matin. Les nouveaux arrivants, parfois contraints de dormir dans la rue, viennent là pour maintenir le lien avec un groupe fluctuant de migrants palestiniens.

Hannerz qualifie de vie enclavée ce mode d'existence urbaine qui se caractérise par " la densité d'un seul secteur du réseau individuel et le fait que cette densité correspond à un ou plusieurs rôles dans lesquels le sujet investit la majeure partie de son temps et de son intérêt " (1982: 316).

Ces jeunes palestiniens ne peuvent compter que sur eux-mêmes pour s'insérer dans la ville. L'association des Palestiniens de Grèce, dont les membres sont venus étudier dans les années 1970 et 1980 et sont aujourd'hui parfaitement intégrés, ne peut que fournir des aides minimes. D'autant plus que l'association est maintenant particulièrement sollicitée par l'afflux de réfugiés Palestiniens en provenance de Syrie depuis le déclenchement du conflit en 2011. De dimension modeste et constituée de notables ayant obtenu la nationalité grecque, elle est loin de disposer des mêmes ressources que ses homologues bangladaises. La présence bangladaise est du reste bien plus importante, notamment autour de la place Omonia, l'un des principaux lieux de centralité migrante d'Athènes où I'on trouve de nombreux magasins et lieux de rassemblement communautaire (Noussia et Lyons, 2009). À la différence de la communauté palestinienne ancienne bien enracinée, les associations de Bangladais se sont constituées en migration et pour la migration.

Aussi les jeunes se trouvent-ils démunis, pris au piège de la ville lointaine. Ces réfugiés-migrants répondent alors moins à la définition de l'homme cosmopolite décrit de façon optimiste par Park et Simmel qu'à I'homme marginal instable et inadapté que définit Stonequist (Cuche, 2009). La position de repli 
que manifeste le retour au camp témoigne de l'échec d'une quête au cours de laquelle " le migrant [...] se présente comme un être intermédiaire, transitoire, hésitant dans le passage des mondes, faisant douloureusement la conquête d'attaches et de la confiance dans les sociétés d'accueil " (Bulle, 2012 : 231-232).

C'est bien le constat lucide que fait Jihad arrivé il y a quelques années de Nahr al-Bared et qui depuis lutte quotidiennement pour survivre à Athènes :

"Regarde, ceux qui sont originaires du Liban [les Palestiniens], ceux qui sont venus et qui sont retournés au Liban. [...] Ils sont rentrés, ils trouvent que la vie est meilleure là-bas. Ici il n'y a pas de vie, ici on meurt de mille morts pour travailler; si tu ne trouves pas de travail, tu vas devoir voler pour régler la location de ton appartement, ou alors tu dors dans une ruine, des ruines, une vieille maison [...] personne n'aura plus jamais de tes nouvelles [...]".

\section{Conclusion : de refuges en migrations}

La ligne suivie dans cet article était d'observer les dimensions relationnelles des passages de trois types de frontières qui sont à la fois territoriales, sociales et culturelles. Ce sont les barrages de l'armée libanaise à l'entrée d'un camp de réfugiés, les routes clandestines vers la Grèce et les relations avec certains des habitants de la capitale de ce pays. Autant de passages problématiques au cours desquels les réfugiés-migrants sont amenés à s'ajuster à la situation, dans le cours d'interactions que l'on peut qualifier d'asymétriques : échanges avec un soldat, un passeur, des gardes-frontières ou encore des citoyens grecs ; seules les sociabilités minimales - réduites à de petites coopérations portant sur I'organisation de la traversée ou sur l'aide à apporter aux femmes et aux enfants lors des embarquements et des débarquements - avec d'autres migrants lors des passages clandestins vers la Grèce se déroulent sur un mode à peu près égalitaire.

La notion d'étrangeté est mobilisée de façon récurrente pour décrire les passages et donner du sens à la situation ; elle en compense d'une certaine manière le caractère outrageant. Notion d'ordre existentielle et non pas causale, car elle décrit sans pour autant expliquer, l'étrangeté renvoie au constat de ne plus avoir prise sur le destin collectif et d'en avoir peu sur sa propre trajectoire. Pourtant la qualification d'étrangeté permet " d'euphémiser " I'hostilité que les représentants légitimes des ordres politiques et/ou urbains peuvent manifester à I'occasion des passages. Le terme traduit un déplacement de la relation de pouvoir, et la catégorisation qui l'accompagne, dans le domaine du non familier comme pour marquer l'écart entre ce qui est et ce qui devrait être dans le cadre " universel " des relations entre humains ; ce que soulignent les discours des réfugiés et des personnes stigmatisées en général sur l'égalité supposée entre les humains que nous avons désignée par la rhétorique de l'humanité. L'étrangeté offre ainsi une issue honorable pour penser le monde inégal.

Car au barrage, dans la ville libanaise proche ou dans la ville lointaine, Athènes, les échanges sociaux se font sous la menace d'une assignation identitaire support de la stigmatisation : réfugié palestinien puis migrant indésirable. Les personnes sont dépossédées de la capacité à négocier la présentation d'elles-mêmes dans l'interaction. À l'inverse, elles sont engagées dans un 
processus où les attributs individuels et collectifs sont assignés par un acteur extérieur. De la sorte, les réfugiés-migrants sont conduits à justifier sans cesse leur position (géographique, socio-politique et culturelle) pour répondre aux questions d'ordre sécuritaire et à leur procédure d'identification, pour s'expliquer sur leur façon d'être et de parler ${ }^{18}$, ou bien pour légitimer leur présence dans un lieu, comme le signale la rencontre entre Nizar et le policier qui lui enjoint de quitter la Grèce et de rentrer chez lui.

Cette asymétrie des positions entraîne une asymétrie de la réflexivité sur les moments de passage particulièrement investis émotionnellement et commentés collectivement par les réfugiés palestiniens. Tandis que les acteurs extérieurs, militaires, policiers, citadins grecs ou libanais interrogent quant à eux le passage des autres, ou bien dit autrement, interrogent les autres sur leur passage. L'attention accordée aux plus modestes, signes d'une reconnaissance manifestant une égalité de traitement, témoigne a contrario de cette inégalité structurelle. L'humanité d'un soldat, l'accueil d'un policier grec dans un centre de rétention qui souhaite la " bienvenue ", un échange sympathique avec un habitant d'Athènes, signalent un changement de statut, fût-il réduit à un bref instant.

Néanmoins, les enquêtés rencontrés après leur retour au camp de Nahr al-Bared ont bel et bien échoué dans leur tentative de migration. Ils reconsidèrent alors les critères d'une vie acceptable et investissent le quotidien du camp pour en quelque sorte réparer les souffrances endurées dans le "voyage " et s'assurer ainsi, en investissant l'ordinaire, une certaine sécurité dans l'existence. Partis jeunes hommes, ils reviennent hommes, ce qui se traduit entre autres choses par la fondation d'une famille. Pour autant, les actions entreprises pour obtenir une vie plus digne sont perçues comme légitimes malgré l'échec. Le regard porté sur l'expérience de migration fait alors valoir une éthique de la mobilité : une façon juste et légitime d'agir opposée à l'indignité de la condition et à la criminalisation du déplacement. Le "voyage " répond ainsi dans une visée éthique au sens que Ricœur attribue à cette notion : " la visée de la vie bonne avec et pour autrui dans des institutions justes "(1990:202). Pour les habitants du camp, cette visée éthique comprend à la fois le droit et l'obligation de se déplacer dans le but de prendre place soi et les siens dans la marche du monde et d'accéder à une vie meilleure. Le départ en migration en quête d'un asile plus digne est la conséquence de cette visée, le retour au camp en marque le rétrécissement, la plongée dans le quotidien l'ultime manifestation, le dernier refuge.

18 Les réfugiés palestiniens sont parfois interrogés sur leur origine quand leur accent est repéré dans les villes du Liban. 


\section{Références bibliographiques}

Agier Michel (2012) Temps, espace et politique, éléments d'anthropologie des camps de réfugiés, in Mohamed Kamel Doraï et Nicolas Puig Éds, L'urbanité des marges. Migrants et réfugiés dans les villes du Proche-Orient, Paris/Beyrouth, Éditions Ifpo/Téraèdre, pp. 29-38.

Bulle Sylvaine (2012) Venir à la présence du monde. L'horizon de la migration comme expérience de soi et comme plan de vie, in Mohamed Kamel Doraï et Nicolas Puig Éds, L'urbanité des marges. Migrants et réfugiés dans les villes du Proche-Orient, Paris/Beyrouth, Éditions Ifpo/Téraèdre, pp. 229-236.

Cuche Denys (2009) "L'homme marginal " : une tradition conceptuelle à revisiter pour penser l'individu en diaspora, Revue Européenne des Migrations Internationales, 25 (3), pp. 13-31.

Doraï Mohamed Kamel (2004) Les réfugiés palestiniens en Europe. Complexité des parcours et des espaces migratoires, Revue Européenne des Migrations Internationales, 20 (2), pp. 169-186.

Giddens Antony (1991) Modernity and Self-Identity. Self and Society in the Late Modern Age, Cambridge, Polity Press, 264 p.

Hannerz Ulf (1983) Explorer la ville, Paris, Les éditions de minuit, 418 p.

Noussia Antonia and Lyons Michal (2009) Inhabiting spaces of liminality: migrants in Omonia, Athens, Journal of Ethnic and Migration Studies, 4, pp. 601-624.

Perdigon Sylvain (2010) L'ethnographie à I'heure des martyrs. Histoire, violence, souffrance dans la pratique anthropologique contemporaine, Annales. Histoire, Sciences sociales, 4, pp. 971-996.

Perdigon Sylvain (2008) La corniche des célibataires. L'intimité à l'épreuve du transnationalisme chez les jeunes Palestiniens de Jal al-Baher, Liban-Sud, Les Cahiers de I'Ifpo, 2, [en ligne] consulté le 8 avril 2013. URL : http://books.openedition.org/ifpo/450

Puig Nicolas (2013) Lost in Transition: Ordeals of Passage of Palestinian Refugees in Lebanon, Mediterranean Politics, 18 (3), pp. 394-410.

Puig Nicolas (2012a) Villes intimes. Expériences urbaines des réfugiés palestiniens au Liban, in Mohamed Kamel Doraï et Nicolas Puig Éds, L'urbanité des marges. Migrants et réfugiés dans les villes du Proche-Orient, Paris/Beyrouth, Éditions Ifpo/Téraèdre, pp. 235-256.

Puig Nicolas (2012b) Le voyage en Grèce. Itinéraires allers-retours de Palestiniens réfugiés au Liban, Métro-politiques, [en ligne]. URL : http://www.metropolitiques. eu/Le-voyage-en-Grece-Itineraires.html

Ricœur Paul (1990) Soi-même comme un autre, Paris, Seuil, 424 p.

Schütz Alfred (1987) Le chercheur et le quotidien. Phénoménologie des sciences sociales, Paris, Klincksieck. 286 p.

Simmel Georg (1991) Essai sur la sociologie des sens, in Georg Simmel, Sociologie et épistémologie, Paris, PUF, pp. 223-238.

Streiff-Fénart Jocelyne and Segatti Aurelia (Eds.) (2011) The Challenge of the Threshold. Border Closures and Migration Movements in Africa, Lanham/ Maryland, Lexington Books, 270 p. 


\section{Nicolas Puig}

\section{... Passages de frontières des Palestiniens du Liban : de refuges en migrations}

À partir d'une analyse des pratiques de mobilités des réfugiés palestiniens au Liban, l'auteur décrit les dimensions relationnelles du passage de différentes frontières territoriales, sociales et culturelles. Ce sont les barrages de l'armée libanaise à l'entrée d'un camp de réfugiés, les routes clandestines vers la Grèce et les relations avec les autorités et certains des habitants de la capitale de ce pays. Autant de passages problématiques au cours desquels les réfugiésmigrants sont amenés à s'ajuster à la situation dans le cadre d'interactions que I'on peut qualifier d'asymétriques : échanges avec un soldat, un passeur, des gardes-frontières ou encore des citadins athéniens. L'auteur cherche à répertorier les réponses aux assignations identitaires et aux injonctions à justifier de leur présence que les réfugiés apportent lors de ces passages et à dégager le point de vue qu'ils développent plus généralement sur leur position entre refuges et migrations.

\section{Crossing Borders: Palestinians Refugees in Lebanon from Refugees to Migrants}

Starting from the practices of mobility of Palestinian refugees in Lebanon, the research describes the relational dimension of the numerous situations of crossing territorial, social and cultural borders and boundaries that the refugees have to go through. These borders and boundaries are the checkpoints of the Lebanese army at the entrance of a refugee camp, the clandestine roads toward Greece and the relationship with the citizens of this country when the refugees reach Athena. In these moments, some forms of asymmetric social exchange take place in the folds of mobilities: exchanges with a soldier, a smuggler, some border guards, or citizen from a foreign country. Starting with refugees from the Palestinian Nahr al-Bared camp in Lebanon, the article describes experiences of passage ranging from the local to the transnational scales.

\section{Cruces de fronteras de los palestinos de Líbano: de refugios en migraciones}

A partir de un análisis de las prácticas de movilidades de refugiados palestinos en Líbano, el autor describe las dimensiones relacionales del hecho de cruzar diferentes fronteras territoriales, sociales y culturales, como son las barricadas del ejército libanés en la entrada de un campo de refugiados, las rutas clandestinas hacia Grecia y las relaciones con las autoridades y algunos de los vecinos de la capital de este país. Durante aquellos tránsitos problemáticos, los refugiados-migrantes están obligados a ajustarse a la situación en el marco de interacciones asimétricas: intercambios con un soldado, un "pollero", un guardia fronterizo o inclusive con ciudadanos de Atenas. Entonces el autor intenta describir las respuestas que los refugiados aportan a las asignaciones identitarias y a los Ilamamientos a justificar su presencia en estos cruces. Además, busca sacar a la luz el punto de vista que ellos desarrollan más ampliamente acerca de su postura, entre refugios y migraciones. 